\title{
DISEÑO MECÁNICO Y MANUFACTURA DE UN DISPOSITIVO DE CARGA PARA ESPECÍMENES ENSAYADOS BAJO RÉGIMEN DE CUASI-RELAJACIÓN IN-SITU
}

\author{
L. A. Alvarez ${ }^{1}$, M. F. Carbajal ${ }^{1}$, R. Sánchez ${ }^{1}$, J. Nieto ${ }^{2}$, L. A. Flores ${ }^{1}$. \\ ${ }^{1}$ Escuela Superior de Ingeniería Mecánica y Eléctrica, Unidad Azcapotzalco (alejandro_v3@hotmail.com) \\ ${ }^{2}$ Unidad Profesional Interdisciplinaria en Ingeniería y Tecnologías Avanzadas
}

Resumen. El método de cuasi-relajación, ha encontrado una amplia aplicación en el estudio de procesos elementales de dislocaciones, siendo fundamental en el estudio y análisis de los mecanismos termoactivos, en la determinación de energía de interacción entre las dislocaciones e inclusiones y, esencialmente, en el estudio de la cinética de la fractura. La problemática que prepondera en el método de cuasi-relajación es el desarrollo de un dispositivo que permita estudiar dicho método. Esto debido a que, el proceder del ensayo emplea especímenes masivos y desarrollo de una considerable deformación plástica en el vértice de la grieta-entalladura de manera descomunalmente sensible, la cual restringe eventualmente este método de estudio. Son muchos los trabajos dedicados al estudio del método descrito, obteniendo información cuantitativa confiable sobre el comportamiento de las dislocaciones partiendo de un modelo analítico del proceso, sin embargo estos trabajos están limitados en el campo experimental, al no contar con una herramienta formal que permita una amplia validación de los modelos analíticos-numéricos, en magnitudes menores a los $5 \AA$, tales experimentos son vítales dentro del campo de la ingeniería de materiales, nanociencias y microscopía. El presente trabajo plasma el desarrollo del diseño mecánico de un dispositivo o mini máquina, acotado en el dispositivo de carga (DC) para ensayos bajo régimen de cuasi-relajación. Para su construcción, se empleó software paramétrico $C A D$ 3D, simulación numérica en software especializado en Análisis por Método de Elemento Finito, todo con el propósito de obtener un modelo que cumpla con las necesidades para realizar el ensayo bajo las condiciones anteriormente mencionadas, con esto, se garantiza que el dispositivo mantenga su posición perfecta en el momento justo de la inmovilización del espécimen durante el ensayo, observando disipaciones de energía, puntos críticos de esfuerzos, estaticidad y dinamicidad del sistema. Finalmente se procedió a su manufactura en un centro de maquinado, ensamblando perfectamente. Usando software de diseño paramétrico CAD/CAM/CAE y poderosos renders, se redujo drásticamente los costos erogados del proceso de fabricación, incluyendo ahorro de tempo vital y prototipos físicos.

Palabras clave: cuasi-relajación, método de elemento finito, manufactura, discretizado. 


\section{INTRODUCCIÓN.}

Partiendo de la curva esfuerzo-deformación de un material típico de ingeniería, la cual presenta las propiedades mecánicas del comportamiento de un material bajo la aplicación de fuerzas externas [1], si bien es fundamental para el diseño estándar en ingeniería, no presenta información más allá del material sometido en algunas condiciones de esfuerzos externos. En ingeniería aplicada, a niveles de red cristalina de un material existen ciertos fenómenos gestados por una vasta cantidad de esfuerzos externos, los cuales suelen ser fulminantes en desarrollo de un defecto fatal en el material.

El método de cuasi-relajación, -generalmente conocido como método de relajación del esfuerzo- ha encontrado amplia aplicación en el estudio de procesos elementales dislocacionales y siendo fundamental en el estudio y análisis de los mecanismos termoactivos, en la determinación de la energía de interacción entre las dislocaciones e inclusiones, y esencialmente, en el estudio de la cinética de la fractura.

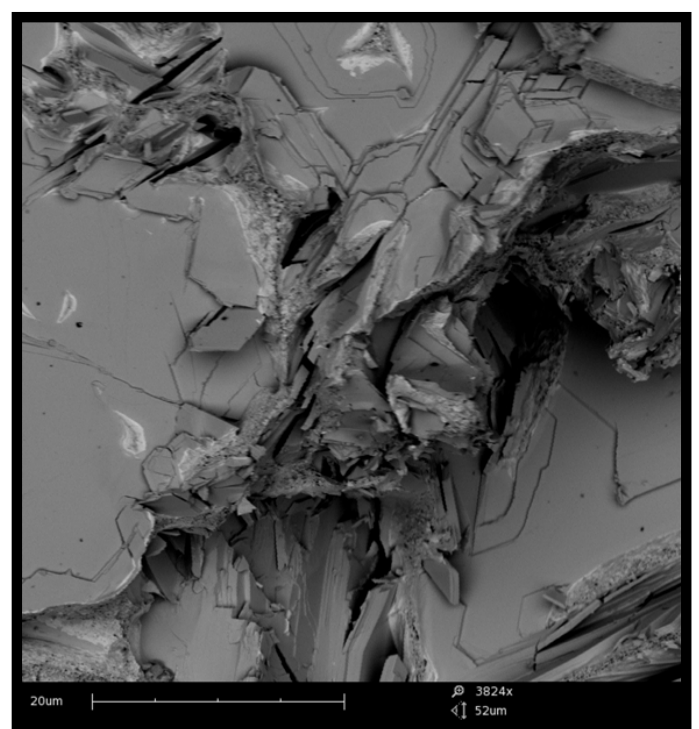

Fig. 1. Ampliación de fractura ocurrida en una fibra de polímero.

La problemática que prepondera en el método de cuasi-relajación es el desarrollo de un dispositivo que permita estudiar éste método. Esto debido a que, el proceder del ensayo emplea especímenes masivos y desarrollo de una considerable deformación plástica en el vértice de la grieta-entalladura de manera descomunalmente sensible, la cual restringe eventualmente este método de estudio [2]. Son muchos los trabajos dedicados al análisis de determinados aspectos teóricos del proceso de fractura, y a la obtención información cuantitativa confiable sobre el comportamiento de las dislocaciones partiendo de un modelo analítico del proceso, y su impacto sobre la forma y el momento cuando se presentan las condiciones para la fractura catastrófica final, sin 
embargo estos trabajos están limitados en el campo experimental, al no contar con una herramienta formal que permita la validación de los modelos analíticos-numéricos.

Ahora bien, es necesario establecer el método ideal que permita efectuar el ensayo bajo régimen de cuasi-relajación y ensayos mecánicos experimentales in-situ, y visualizar el proceso evolutivo de agrietamiento y relajación en tiempo real en magnitudes menores a los $5 \AA$, experimentos vítales dentro del campo de la ingeniería de materiales, nanociencias y microscopía.

El método ideal para efectuar dicho ensayo es, apoyarse en la microscopía electrónica, puntualizando el uso del SEM y otros microscopios electrónicos, dadas las características físicas de éstos instrumentos, es posible desarrollar un dispositivo que pueda ser alojado dentro de la columna o espacio de trabajo de ellos, y ejecutar pruebas mecánicas elementales, tales como ensayos de tensión, compresión, torsión y flexión. El dispositivo en sí, deberá ser una máquina de tracción en miniatura destinada a la ejecución de ensayos mecánicos, accionado por un sistema de electromotores que permiten deformar micro-especímenes bajo un régimen de velocidad constante de carga hasta llevarlo a la fractura, todo esto directamente dentro de la columna del SEM, HRTEM, FIB, etc.

Una vez establecido esto, se diseñan las probetas de los especímenes a ensayar, estos podrán ser de cualquier material, -en caso de ser no conductores, se recubren de una película de oro u otro material conductor- dichas probetas son diseñadas bajo las normas vigentes para ensayos y pruebas mecánicas (ASTM-E4)[3]. Las probetas serán sujetadas por un par de mordazas, una vez instalada la probeta con las mordazas, estás son colocadas dentro del SEM y posteriormente se cierra su puerta de acceso a la columna, se orienta a la probeta con el emisor de electrones del microscopio y finalmente se energiza el SEM para comenzar con el barrido de la superficie establecida del espécimen.

Ahora se debe energizar el dispositivo para ensayos, -es muy importante mencionar que la velocidad de desplazamiento de las mordazas deberá ser extremadamente pequeña, ya que las dimensiones a las que se está siendo efectuando el ensayo es del orden menor a $5 \AA$ A - al energizar, se deberá tener un sistema que controle perfectamente el instante en que se va iniciar el ensayo, por ejemplo, si fuera un ensayo a tracción, se deberá tener control total sobre los instantes en que comenzará a desplazarse la mordaza y tensará al espécimen así como al momento de pausar el ensayo. Una vez comenzado el ensayo, en un intervalo de tiempo muy breve se deberá detener el tensado del espécimen, -como anteriormente se ha expuesto, esto deberá ser en diversos puntos del material, en intervalos de comportamiento elástico y comportamiento plástico- dejando transcurrir un intervalo de tiempo "t(x)", este lapso “ $\mathrm{x}$ ” de tiempo favorecerá a la relajación del esfuerzo, para una vez transcurrido el lapso de tiempo volver a reanudar el ensayo, esto una fracción de distancia, y análogamente se realizará este proceso hasta el punto de fractura del material. Con ello se estudiarán los mecanismos de cinética de red y procesos de dislocaciones del material en diversos puntos de su curva esfuerzo-deformación hasta el proceso catastrófico de fractura.

Para que este ensayo pueda ser confiable, los elementos que integran al dispositivo de ensayos, no deberá de presentar deformación algunas durante los ensayos, tampoco pérdida de energía de ningún tipo y ser excelentemente estático al momento de pausar y mantenerse así du- 
rante el tiempo que sea necesario, así mismo ser excelentemente dinámico al momento de reanudar el ensayo, esto es, que no presente ningún tipo de sobre compensación cuando se reanude el movimiento de las mordazas, y que el más pequeño desplazamiento o perturbación de alguno de los dispositivos afectarán el ensayo.

\section{METODOLOGÍA DE DISEÑO.}

La metodología de diseño empleada en el desarrollo del proyecto, ha sido desarrollada por un grupo de universidades de Latinoamérica, tales como la Universidad Simón Bolívar de Venezuela y el Instituto Politécnico Nacional de México, los resultados que se han obtenido al emplear dicha metodología fueron favorables en los casos de aplicación. La metodología consiste en cuatro principales fases, en las que a su vez son sub divididas en grupos jerárquicos, hasta completar las cuatro fases. Durante el desarrollo de esta metodología, existe la retroalimentación entre cada grupo jerárquico de cada fase. En la Figura 2, se observa el desarrollo de esta metodología de diseño.

\section{OBJETIVO DE DISEÑO DEL DISPOSITIVO DE CARGA (DC).}

El diseño de la nueva máquina que será alojada en el microscopio electrónico está divida en cuatro etapas:

1. Diseño de soporte y mordazas (dispositivo de carga),

2. Diseño del sistema de ajuste y orientación en el plano $X Z$,

3. Sistema de potencia de la máquina,

4. Sistema de control y adquisición de datos. (Todo en dimensiones reducidas)

Un punto importante a mencionar, es la velocidad del desplazamiento de las mordazas, esta va directamente ligada al torque y velocidad del motor y los elementos mecánicos como las transmisiones y tornillos de potencia, la velocidad que maneja ésta máquina es de $0.05 \mathrm{~mm}$ por minuto.

Algunas características importantes del DC:

- Máxima carga aplicable: $250 \mathrm{~kg}$.

- Velocidad de desplazamiento de la mordaza móvil, $0.05 \mathrm{~mm} / \mathrm{min} ; 0.1 \mathrm{~mm} / \mathrm{min}$;

\section{$0.2 \mathrm{~mm} / \mathrm{min}$ y $0.4 \mathrm{~mm} / \mathrm{min}$.}

- Sección transversal de los especímenes ensayados: 3x1 $\mathrm{mm}^{2}$.

- Máximo desplazamiento de la mordaza móvil: 30mm.

- Desplazamiento máximo en dirección perpendicular al eje del espécimen: +3mm.

- El dispositivo debe garantizar la deformación de los micro-especímenes tanto en régimen de tracción como en flexión. 


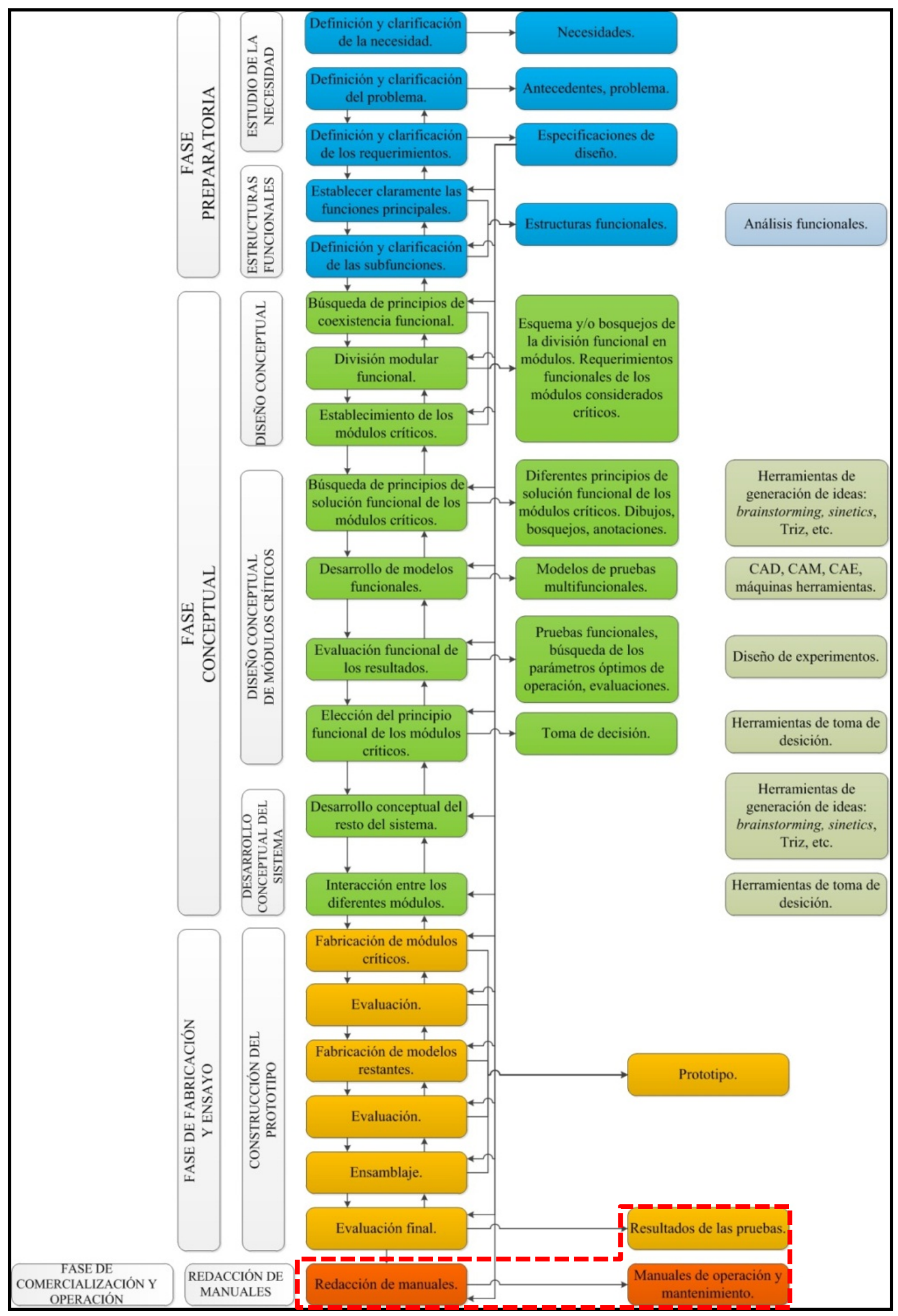

Figura 2. Diagrama de la metodología de diseño empleada para el desarrollo de la máquina de ensayos, la parte entre líneas punteadas indica la fase que falta por desarrollar. 


\section{EL DISPOSITIVO DE CARGA (DC)}

El dispositivo de carga está destinado para la ejecución de experimentos de deformación de micro-especímenes directamente dentro de la columna del microscopio electrónico de barrido $S E M$, bajo condiciones de carga cuando se efectúa un registro continuo de la deformación y del esfuerzo, que actúan sobre la parte de trabajo del espécimen y con una permanente visualización de las variaciones estructurales que operan sobre su superficie, es decir, para la ejecución de ensayos in-situ de deformación dentro de la columna del SEM.

El dispositivo de carga, DC, garantiza:

1. Deformar bajo condiciones de tensión micro-especímenes fabricados en materiales conductores, con una carga máxima en tensión de $200 \mathrm{~kg}$; ésta es una condición suficiente para fracturar micro-especímenes fabricados en metales de resistencia media, con un límite de ruptura de $\sigma_{r}=600 \mathrm{MPa}$, y con una superficie de sección del micro-espécimen de 3m² .

2. Deformar micro-especímenes bajo condiciones de flexión en tres puntos, con una sección de 4 X $5 \mathrm{~mm}^{2}$.

Es importante señalar que, siendo necesario el estudio del comportamiento mecánico de materiales no conductores, se requiere, con el objetivo de evitar ruptura y distorsiones en las imágenes, recubrir mediante pulverización por plasma con metales las superficies de los especímenes. En la Figura 3, se observa el modelo final del dispositivo de carga, donde, en el sistema de mordazas está montada una probeta de cobre de $3 \mathrm{~mm}^{2}$ en su sección transversal. En la Figura 4, (parte superior), se observa los cortes correspondientes a los planos horizontales y verticales, lo cuales permiten una visualización de los componentes y estructura interna de los mismos. Esto con la finalidad de permitir un mejor entendimiento sobre la constitución del DC. En la Figura 4, (parte inferior) están mencionados los componentes principales de este DC, destacando el sistema de mordazas, así como el sistema eje-tornillo.

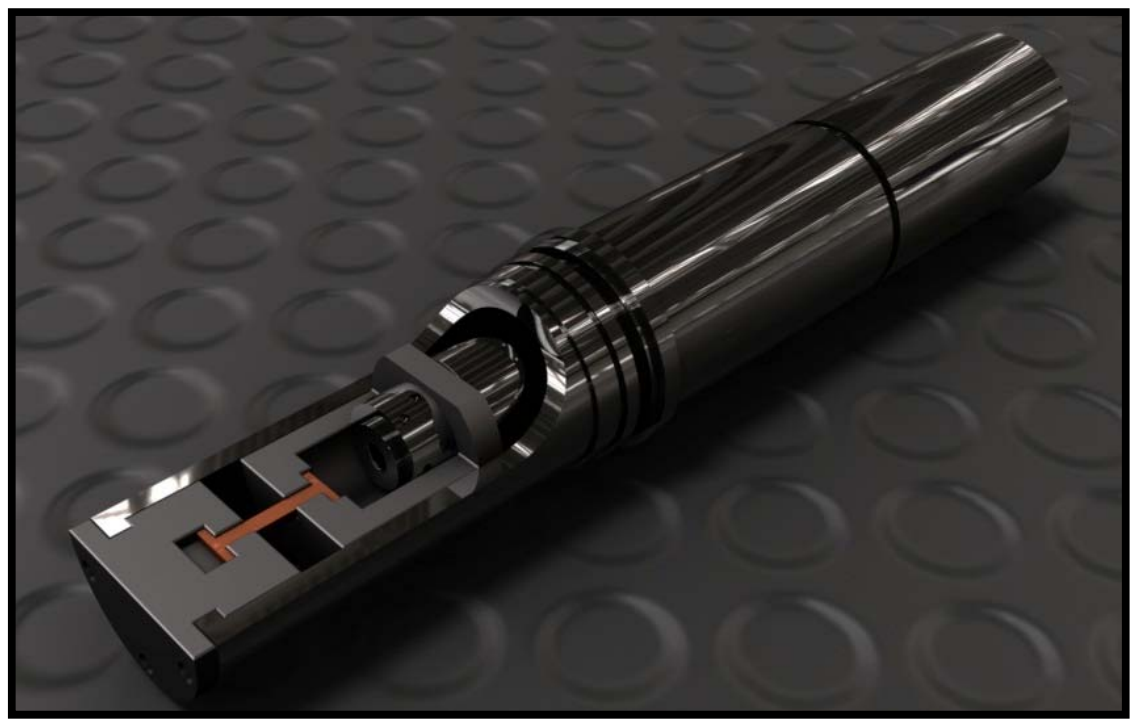

Figura 3. Modelo final del dispositivo de carga DC en acero inoxidable. 
El conjunto completo del dispositivo de carga está constituido por un cuerpo sobre el cual está montado el sistema electromecánico que consiste en un transmisor de engranajes y tornillos sin fin, que ejecuta el desplazamiento de la mordaza móvil del espécimen con una velocidad constante y predeterminada, una caja de velocidades que permite efectuar las reducciones en el número de revoluciones del eje del sistema electromotor, con una relación de transmisión fija de dos diferentes relaciones y conexión del sistema manual de movimiento de la mordaza.

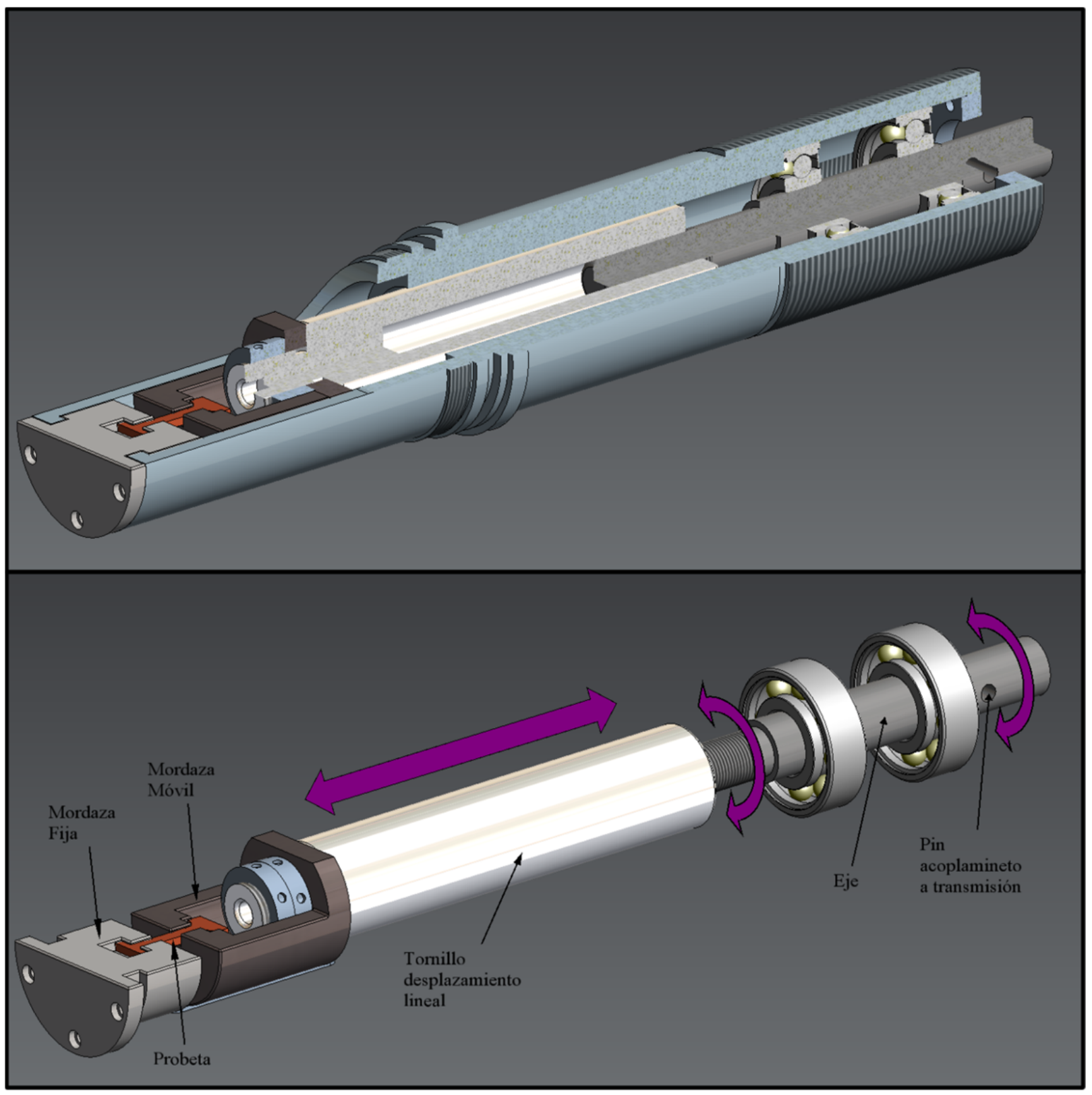

Figura 4 (Superior). Corte al dispositivo de carga, permitiendo la visualización de los componentes internos. Figura 4 (Inferior). Mención de los componentes principales del dispositivo de carga, el eje está conectado por medio de un perno a la transmisión, la cual a su vez está acoplado al electromotor, en donde se inicia el movimiento. 
El electromotor cuenta con sistema de reducción integrado de diferentes relaciones de transmisión, un sensor de registro de fuerza, montado sobre la base de la mordaza móvil, un mecanismo de regulación de la posición espacial del micro-espécimen en tres coordenadas, un sistema hermético que garantiza el vacío del dispositivo que comprende un sistema de sellos de vacío, un espiral flexible y una compuerta especial agregada a la cámara de vacío del microscopio y un sistema integrado por la mordaza fija.

\section{MODELO ANALÍTICO DEL SISTEMA TORSOR Y DEL SISTEMA EN TRES DI- MENSIONES.}

Primero, el modelo general que describe al sistema de ecuaciones está dado por

$$
[\boldsymbol{K}]\{\boldsymbol{\phi}\}=\{\boldsymbol{F}\}
$$

Lo que se puede generalizar en la siguiente expresión

$$
[\boldsymbol{K}]\{\boldsymbol{\phi}\}-\{\boldsymbol{F}\}=\{\boldsymbol{R}\}
$$

Donde $\{\boldsymbol{R}\}$ es la matriz de reacción. Ahora para el modelado en tres dimensiones [4] queda expresado de la siguiente forma

$$
\frac{\partial}{\partial x}\left[\boldsymbol{k}(\boldsymbol{\phi}) \frac{\partial \boldsymbol{\phi}}{\partial x}\right]+\frac{\partial}{\partial y}\left[\boldsymbol{k}(\boldsymbol{\phi}) \frac{\partial \boldsymbol{\phi}}{\partial y}\right]=-\boldsymbol{Q}(x, y)
$$

Aplicando el método de diferenciación finita, la ecuación resultante es

$$
\begin{gathered}
\frac{\left[-\boldsymbol{k}_{l+\frac{1}{2}, m} \boldsymbol{\phi}_{l+1, m}+\left(\boldsymbol{k}_{l+\frac{1}{2}, m}+\boldsymbol{k}_{l-\frac{1}{2}, m}\right) \boldsymbol{\phi}_{l, m}-k_{l-\frac{1}{2}, m} \boldsymbol{\phi}_{l-1, m}\right]}{\Delta x^{2}} \\
+\frac{\left[-\boldsymbol{k}_{l+\frac{1}{2}, m} \boldsymbol{\phi}_{l, m+1}+\left(\boldsymbol{k}_{l, m+\frac{1}{2}}+\boldsymbol{k}_{l, m-\frac{1}{2}}\right) \boldsymbol{\phi}_{l, m}-\boldsymbol{k}_{l, m-\frac{1}{2}} \boldsymbol{\phi}_{l, m-1}\right]}{\Delta y^{2}} \\
l=1,2, \ldots, L-1 ; m=1,2, \ldots, M-1
\end{gathered}
$$

Para la rotación del eje, considerando que el eje está fijo respecto a su eje de sección transversal, para así transmitir la energía a lo largo del sistema [5]. El sistema de coordenadas está integrado en un punto fijo y de rotación. El vector sin deformación, $\boldsymbol{X}^{\prime}$ en el sistema de rotación esta relacionado a su sistema homólogo, $\boldsymbol{X}$, en el sistema sin rotación descrito por $\boldsymbol{X}^{\prime}=\boldsymbol{Q}(t) \boldsymbol{X}$. La parte homóloga para la deformación de la posición es $\boldsymbol{x}^{\prime}=\boldsymbol{Q}(t) \boldsymbol{x}$. El desplazamiento también 
satisface $\boldsymbol{u}^{\prime}=\boldsymbol{Q}(t) \boldsymbol{u}$. La rotación esta representada por el vector axial $\boldsymbol{\omega}$, satisfaciendo $\boldsymbol{\omega} \times$ $(*)=\dot{\boldsymbol{Q}}(t) \boldsymbol{Q}^{\boldsymbol{T}}(t)(*)$.

Al tiempo que la derivada del eje de rotación satisface

$$
\begin{gathered}
\frac{d \boldsymbol{u}^{\prime}}{d t}=\frac{\partial \boldsymbol{u}^{\prime}}{\partial t}+\boldsymbol{\omega} \times \boldsymbol{u}^{\prime} \\
\frac{\partial^{2} \boldsymbol{u}^{\prime}}{d t^{2}}=\frac{\partial^{2} \boldsymbol{u}^{\prime}}{d t^{2}}+2 \boldsymbol{\omega} \times \boldsymbol{u}^{\prime}+\boldsymbol{\omega} \times \boldsymbol{\omega} \times \boldsymbol{u}+\boldsymbol{\alpha} \times \boldsymbol{u}
\end{gathered}
$$

Donde $\boldsymbol{\alpha}=\partial \boldsymbol{\omega} / \partial t$ y $\partial(.) / \partial t$ implica diferenciación con el sistema de coordenadas instantáneo fijo. Los cuatro términos más a la derecha son llamados translaciones, Coriolis, centrífuga, y aceleración angular, respectivamente.

Aplicando el principio del Trabajo Virtual, los términos inerciales se transforman $\int \delta \boldsymbol{u}^{\prime T} \rho d^{2} / d t\left[\boldsymbol{u}^{\prime}+\boldsymbol{X}^{\prime}\right] d V$. Asumiendo que $\boldsymbol{u}^{\prime}=\boldsymbol{\varphi}^{T}\left(\boldsymbol{X}^{\prime}\right) \boldsymbol{\Phi} \boldsymbol{\gamma}(t)$,

$$
\int \delta \boldsymbol{u}^{\prime \boldsymbol{T}} \rho \frac{d^{2}}{d t}\left[\boldsymbol{u}^{\prime}+\boldsymbol{X}^{\prime}\right] d V=\delta \boldsymbol{\gamma}^{\boldsymbol{T}}\left[\boldsymbol{M} \frac{d^{2} \boldsymbol{\gamma}}{d t^{2}}+\boldsymbol{G}_{\mathbf{1}} \frac{d \boldsymbol{\gamma}}{d t}+\left(\boldsymbol{G}_{\mathbf{2}}+\boldsymbol{A}\right) \boldsymbol{\gamma}\right]
$$

Donde

$$
\begin{gathered}
\boldsymbol{M}=\boldsymbol{\Phi}^{T} \int \rho \boldsymbol{\varphi} \boldsymbol{\varphi}^{T} d V \boldsymbol{\Phi} \\
\boldsymbol{G}_{1}=\boldsymbol{\Phi}^{T} \int \rho \boldsymbol{\varphi} \boldsymbol{\Omega} \boldsymbol{\varphi}^{T} d V \boldsymbol{\Phi} \\
\boldsymbol{G}_{2}=\boldsymbol{\Phi}^{T} \int \rho \boldsymbol{\varphi} \boldsymbol{\Omega}^{2} \boldsymbol{\varphi}^{T} d V \boldsymbol{\Phi} \\
\boldsymbol{A}=\boldsymbol{\Phi}^{T} \int \rho \boldsymbol{\varphi} \mathbf{A} \boldsymbol{\varphi}^{T} d V \boldsymbol{\Phi}
\end{gathered}
$$

La matriz $\boldsymbol{M}$ es por convención definida-positiva y matriz de masa simétrica; la matriz de Coriolis $\boldsymbol{G}_{\mathbf{1}}$ es antisimétrica; la matriz de aceleración centrífuga $\boldsymbol{G}_{\mathbf{2}}$ es definida-negativa; y la matriz de aceleración angular $\boldsymbol{A}$ es antisimétrica [5].

\section{ANÁLISIS POR MÉTODO DE ELEMENTO FINITO DEL D.C.}

Antes de comenzar con la manufactura del Dispositivo de Carga, se efectúa la validación del modelo matemático, empleando Software computacional especializado en la resolución de problemas de ingeniería mediante el Análisis por Método de Elemento Finito (FEA).

El desarrollo del análisis numérico se divide en varias fases o etapas. En la Figura 5, se muestra el diagrama que hace mención a los pasos utilizados para el desarrollo del dispositivo de carga, en él se muestra desde que se comienza con el bosquejo hasta la culminación con la manufactura del dispositivo de carga. 
En el análisis numérico, ya teniendo el modelo CAD en 3D, se exporta al Software FEA. Una vez importada la geometría, se discretiza (mallado) el modelo, sabiendo que, en función de la calidad del discretizado estará la convergencia de los resultados, esto es, si es más denso el discretizado, mayor será la aproximación a una solución favorable del modelo, por ello se debe considerar si el discretizado obtenido es óptimo o no para el análisis final. En este caso, los discretizados obtenidos tuvieron un refinamiento de $5 \mathrm{u}$, esto debido a que las dimensiones son extremadamente pequeñas $(\mu)$, lo cual requiere que el análisis sea muy preciso. Posteriormente, en los resultados se observará si efectivamente el diseño es el correcto o debe hacerse una corrección en él. Para ello, se analizan los valores de deformación, desplazamiento y energía disipada. Algo muy importante a tener en cuenta, es, entre más densa sea un discretizado, mayor tiempo de procesamiento y utilización de recursos computacionales se requerirán para resolver el modelo.

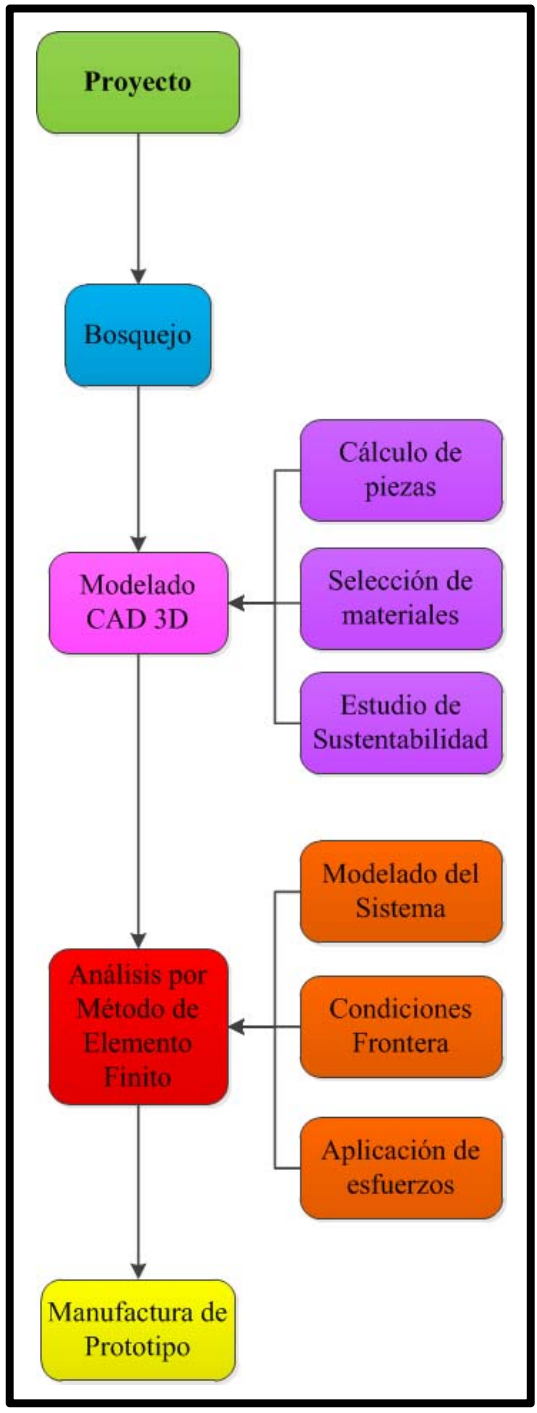

Figura 5. Diagrama del desarrollo del dispositivo de carga, así como las actividades que integran cada etapa del diseño. 
En la parte numérica, se muestran tres análisis obtenidos con el software, la Figura 6 corresponde al modelado y análisis del eje que se acopla a la transmisión, el modelado en CAD (Figura 6a) está acotado en micras $(\mu)$ para facilitar la interpretación de los resultados y evitar el uso de conversiones, el modelo fue hecho en 3-D mediante sólidos en revolución. Una vez generado el modelo, éste fue exportado el software de análisis por elemento finito, en el cual se comenzó por generar el discretizado, para esta parte, se dejó que el software realizará el mallado de forma automática, una vez que se termino con la operación de mallado, se inspeccionó la malla creada, esto para determinar si el mallado obtenido permitiría una correcta convergencia de resultados, al hacer la inspección se observaron secciones que carecían de los suficientes elementos como para obtener un resultado preciso, por ello, se refino la malla en un factor de 5, esto se hizo de forma manual, seleccionando las regiones donde se necesitaría un refinamiento mayor.

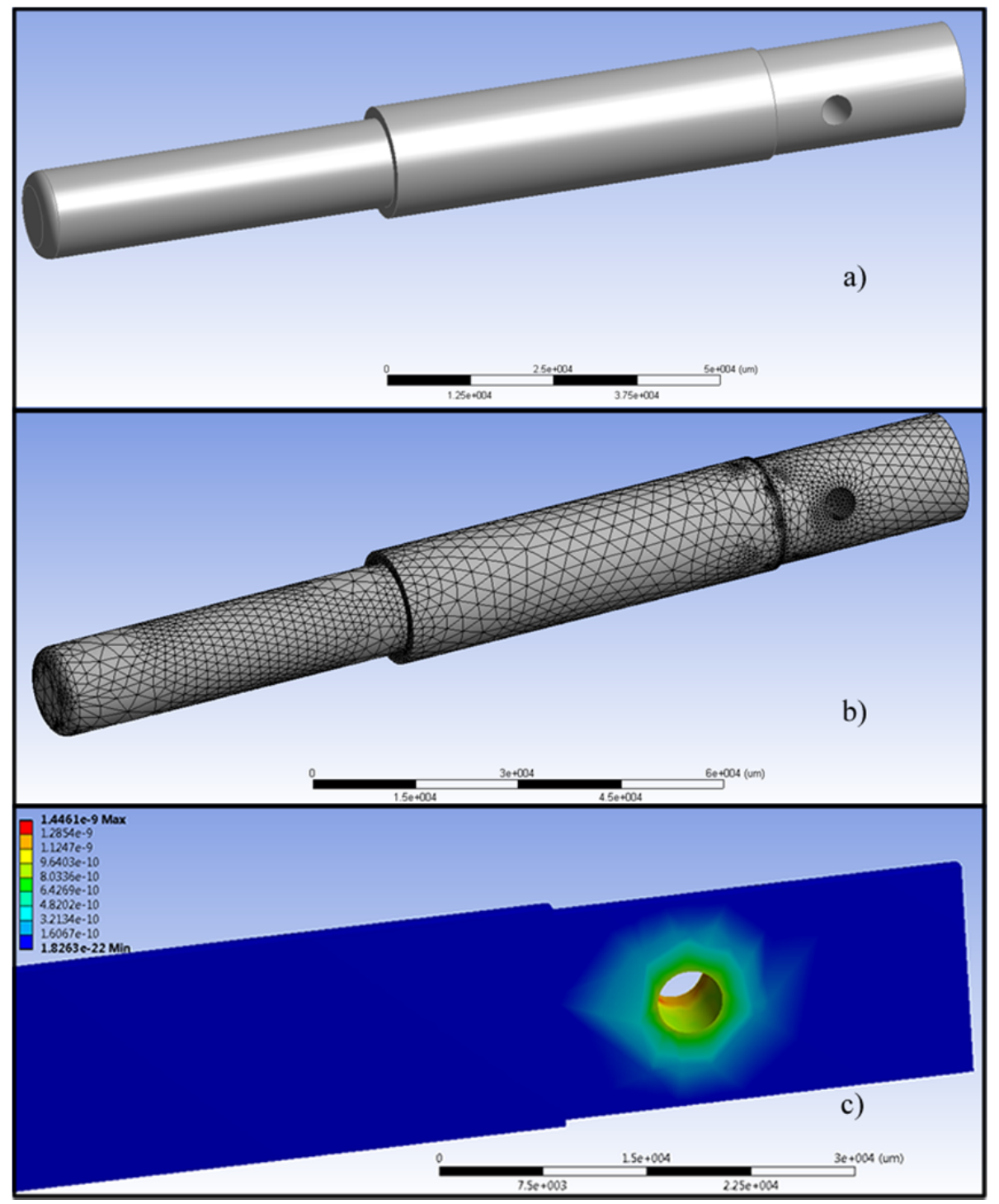

Figura 6. Imágenes del modelo y análisis numérico del eje, así como del esfuerzo generado en el pin del eje. 
El resultado final del mallado se observa en la Figura 6b. Ahora, en la Tabla 1, se presentan lo datos obtenidos en el mallado del eje, así como otros aspectos relevantes obtenidos durante el mallado. El material empleado fue acero inoxidable T-304.

Tabla 1. Datos generales en el mallado del eje.

\begin{tabular}{lr}
\hline \multicolumn{2}{c}{ Mallado Estático } \\
\hline Material & T-304 \\
Nodos generados & 37972 \\
Elementos generados & 23782 \\
Áreas refinadas & 5 \\
Factor de refinamiento & 5 \\
\hline
\end{tabular}

La Figura 6c, se muestra en análisis que represente la torsión máxima y mínima que se genera al aplicar la carga equivalente necesaria para generar una ruptura en la probeta, como es observable, el esfuerzo generado queda muy por debajo del límite permisible del acero inoxidable. Por ello se determina que el modelo propuesto del eje es el idóneo para el dispositivo de carga. De forma similar también se obtuvieron los resultado para la energía total absorbida por el elemento, la deformación total ocurrida, esto en diversos instantes de tiempo, para 0s, 5s, 10, 40s, 80s, 120s, 180s, y 300s, siendo 0s, el instante en que se comienza con el análisis, y 300s, el instante donde se terminar el análisis, momento previo a la fractura de la probeta.

En la Figura 7, corresponde al modelado y análisis del tornillo con desplazamiento lineal que va acoplado al eje, analógamente se realiza el mismo procedimiento que en el modelado y análisis del eje. En la Figura 7a y 7b, se observa el modedalo CAD 3D y el mallado del tornillo. En la Tabla 2, se presentan los datos obtenidos durante el mallado.

Tabla 2. Datos generales en el mallado del tornillo.

\begin{tabular}{lr}
\hline \multicolumn{2}{c}{ Mallado Estático } \\
\hline Material & $\mathrm{T}-304$ \\
Nodos generados & 52043 \\
Elementos generados & 30709 \\
Áreas refinadas & 4 \\
Factor de refinamiento & 5 \\
\hline
\end{tabular}

En la Figura 7c, se presenta el desplazamiento generado al aplicar la fuerza necesaria para generar la ruptura en una probeta con límite de ruptura medianamente alto, alrededor de 250 $\mathrm{kg} / \mathrm{mm}^{2}$. El desplazamiento máximo obtenido después de 5 minutos fue de sólo $0.000022 \mu$. Mas adelante se observa que este desplazamiento está directamente transmitido al sistema de mordazas, acumulándose toda la carga en el centro de la probeta.

La energía perdida durante el análisis numérico en los elementos móviles y rígidos fue de $0 \%$, esto significa que el total de la energía es absorbida por la probeta, desde el instante que se comienza con el ensayo, hasta la parte final del mismo, aproximadamente 5 minutos después. 


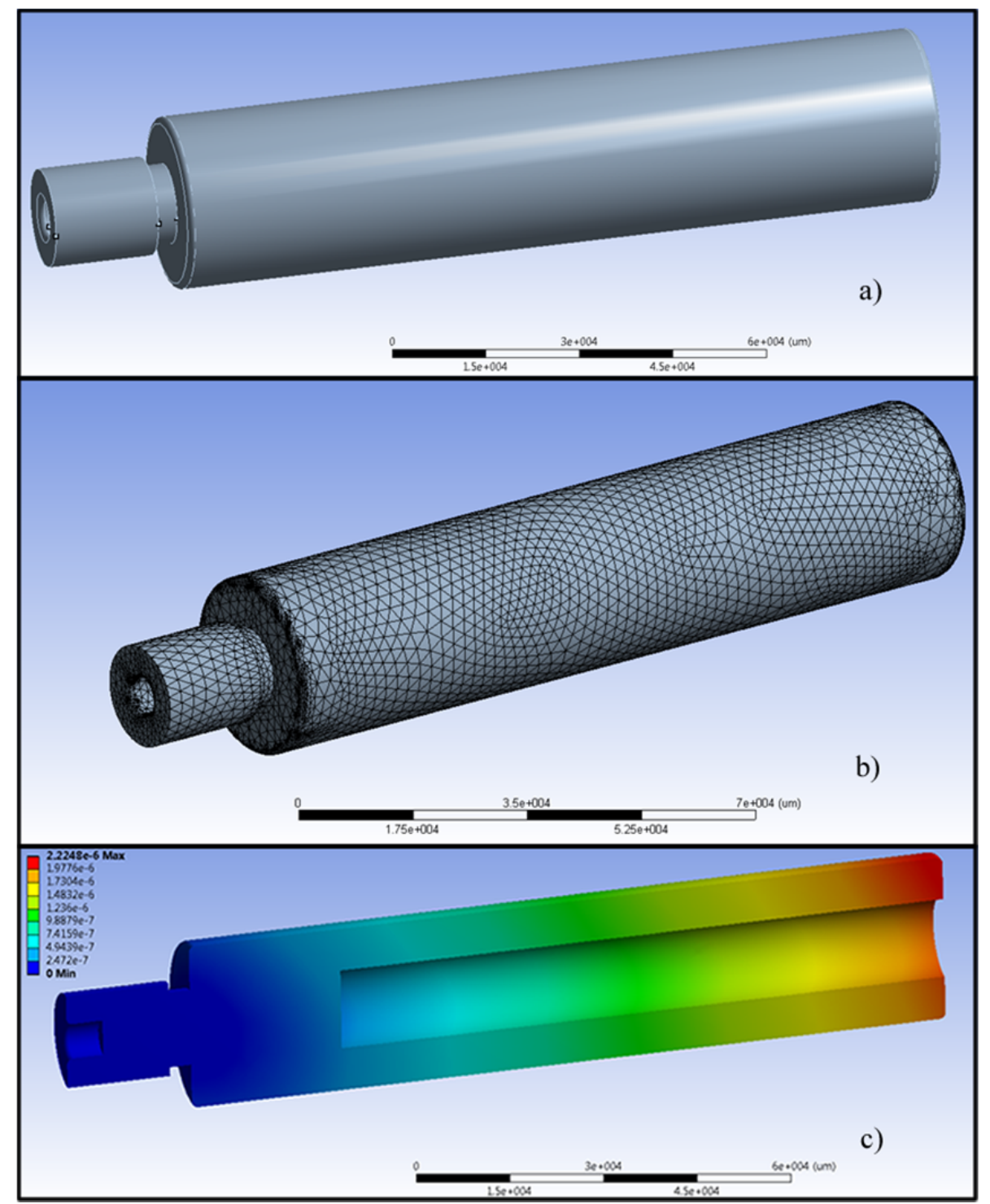

Figura 7. Imagen que muestra el modelado CAD y análisis numérico del tornillo. También se presenta el imagen que muestra el desplazamiento total ocurrido en el momento previo a la fractura de la probeta (Figura 6c).

La última parte que se presenta en este estudio, es la que corresponde al sistema de mordazas. En la Figura 8, se muestran las tres partes que integran al sistema, la mordazas móvil, la mordazas fija y la probeta. En la Tabla 3, se mencionan las características generales empleadas en el análisis numérico.

Tabla 3. Generalidades del análisis del sistema de morzadas. .

\begin{tabular}{ll}
\hline & Sistema de mordazas \\
\hline Material de mordazas & Acero cementado Cr-Ni-Mo \\
Material probeta & Cobre \\
Número total de cuerpos & 3 \\
Número total de cuerpos en contacto & 3 \\
Máxima carga axial aplicada & $250 \mathrm{Kg}$. \\
\hline
\end{tabular}




\begin{tabular}{ll}
\hline Tiempo total de análisis & $300 \mathrm{~s}$. \\
Número de pasos & 8 \\
Número total de áreas & 102 \\
Temperatura & $22^{\circ}$ \\
Presión & $1 \mathrm{~atm}(1.013 \mathrm{bar})$ \\
\hline
\end{tabular}

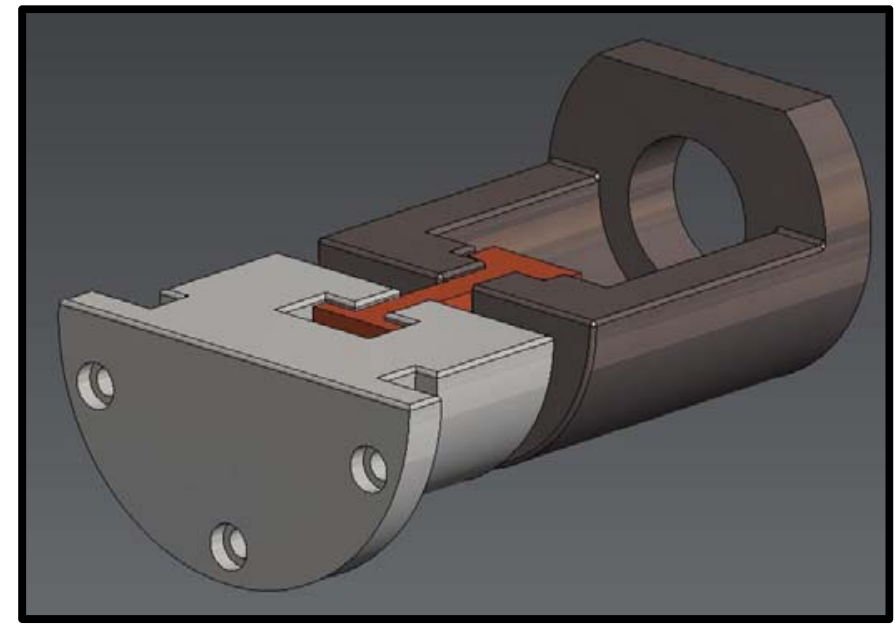

Figura 8. Sistema de mordazas, de izquierda a derecha, mordazas fija, probeta, mordaza móvil.

En la Tabla 4, se muestran las características del mallado del sistema de mordazas, en ella se incluyen los cuerpos totales que conforman al sistema, así como de la probeta, el factor de refinamiento y los nodos y elementos totales generados.

Tabla 4. Datos generales en el mallado del sistema de mordazas.

\begin{tabular}{lc|c|c}
\hline \multicolumn{3}{c}{ Mallado Estático } \\
\hline Cuerpos totales & 3 \\
Nodos generados & 186893 & 219358 & 112976 \\
Elementos generados & 62998 & 78553 & 6841 \\
Áreas refinadas & 24 & \\
Factor de refinamiento & \multicolumn{2}{|c}{5} \\
\hline
\end{tabular}

Para el análisis numérico del sistema de mordazas se hicieron diversas corridas, las cuales arrojaron interesantes resultados, entre ellos se muestran tres de estos resultados, considerando los que aportan una mayor cantidad de información y más clara en el análisis. En la Figura 9, se presentan estos resultados. En la Figura 9a, se observa la energía total absorbida por el sistema, en ella se aprecia que el total de la energía es absorbida por la probeta, y puntualmente esta absorción ocurre en el centro de la probeta, lo cual reafirma el correcto modelo del sistema de mordazas.

La Figura 9b, corresponde al desplazamiento total de la mordaza móvil, el cual es uniformemente transmitido por toda la mordaza móvil hasta la probeta, en ella se comienza la estrangulación de su sección y queda aislada en este punto, evitando micro desplazamientos en la mordaza fija, los cuales pudieran en la práctica causar errores potenciales en la relajación del material. 
Finalmente en la Figura 9c, se observan los máximos y mínimos esfuerzo que se presentan en el sistema de mordazas, igualmente que en las anteriores, la máxima y total concentración de esfuerzos ocurre en la parte central de la probeta, justamente en donde analíticamente se presentará la fractura del material.

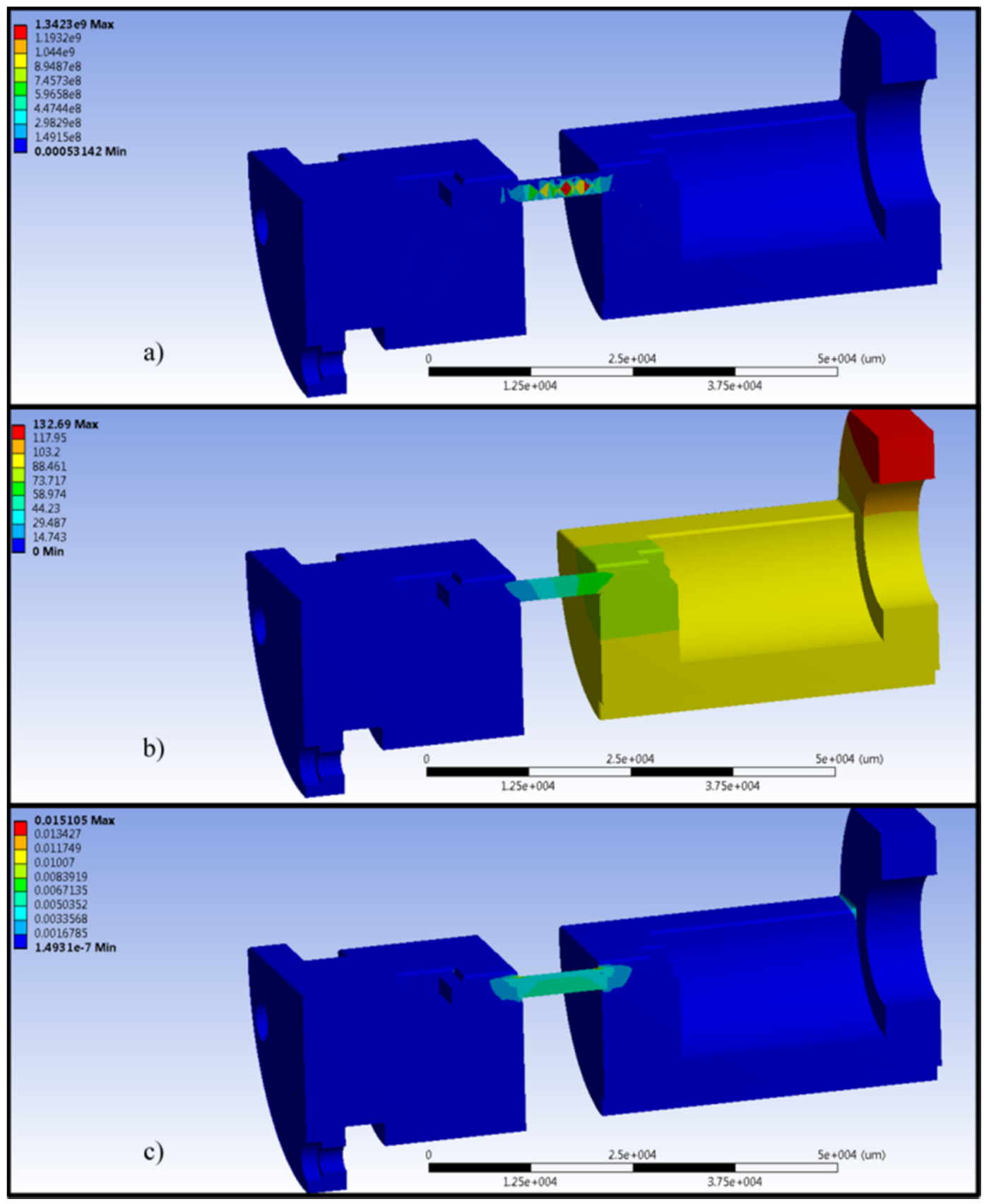

Figura 9. Análisis del sistema de mordazas, en las cuales es evidente la concentración de energía, esfuerzos y el desplazamiento total ocurrido. 


\section{MANUFACTURA Y ENSAMBLAJE DEL DISPOSITIVO DE CARGA}

Para la manufactura del dispositivo de carga se usó un CNC de la marca Centroid ${ }^{\circledR}$ de 5GDL. Una vez concluida la etapa de manufactura de cada componente, se realiza el ensamble de estos, es primordial que los elementos ensamblen correctamente, ya que cualquier defecto en el material o en el maquinado de las piezas proporcionará información no confiable durante el ensayo.

Con el uso de software de diseño paramétrico CAD y CAM se redujo drásticamente los costos erogados del mismo proceso de fabricación, incluyendo ahorro de vital tiempo. Una vez más, este es un claro ejemplo de aplicación de las correctas herramientas para la fabricación integral de un novedoso producto.

La manufactura final y el ensamble de los elementos del dispositivo de carga fue el esperado, apegándose al diseño conceptual previo.

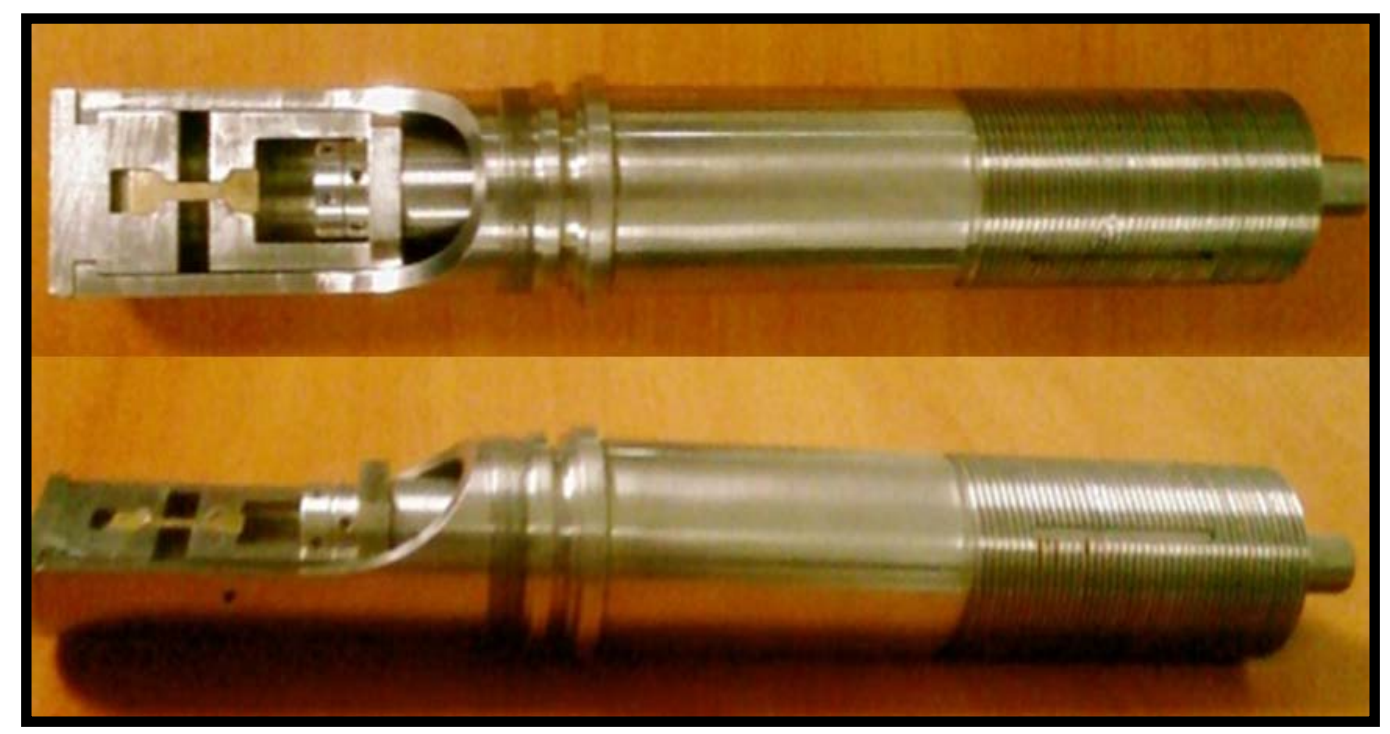

Figura 10. Manufactura del Dispositivo de Carga

El dispositivo de carga está destinado para ser utilizado en la realización experimentos científico-técnicos a nivel de laboratorio en los campos específicos de la física y de la metalurgia, durante la realización de complejos experimentos dirigidos al estudio de las propiedades mecánicas de los materiales, a la determinación de sus propiedades mecánicas y al análisis de las características estructurales que surgen en la zona de trabajo de los especímenes ensayados, como resultado de la deformación plástica. Con la aplicación de ensayos que permitan observar el comportamiento de un material a niveles atómicos bajo ciertas condiciones iniciales, se marca la pauta en la investigación de nuevos fenómenos que hasta hoy no ha sido posible caracterizar ni visualizar en tiempo real, es por ello que es fundamental el desarrollo de este tipo de tecnologías. 


\section{CONCLUSIONES}

Con el uso de software de diseño paramétrico CAD y CAM se redujo drásticamente los costos erogados del mismo proceso de fabricación, incluyendo ahorro de vital tiempo. Una vez más, este es un claro ejemplo de aplicación de las correctas herramientas para la fabricación integral de un novedoso producto.

La manufactura final y el ensamble de los elementos del dispositivo de carga fue el esperado, apegándose al diseño conceptual previo.

El dispositivo de carga está destinado para ser utilizado en la realización experimentos científico-técnicos a nivel de laboratorio en los campos específicos de la física y de la metalurgia, durante la realización de complejos experimentos dirigidos al estudio de las propiedades mecánicas de los materiales, a la determinación de sus propiedades mecánicas y al análisis de las características estructurales que surgen en la zona de trabajo de los especímenes ensayados, como resultado de la deformación plástica. Con la aplicación de ensayos que permitan observar el comportamiento de un material a niveles atómicos bajo ciertas condiciones iniciales, se marca la pauta en la investigación de nuevos fenómenos que hasta hoy no ha sido posible caracterizar ni visualizar en tiempo real, es por ello que es fundamental el desarrollo de este tipo de tecnologías.

La máquina es de costo reducido así como sus dimensiones y su fácil adaptabilidad a nuevos instrumentos usados dentro del campo de la microscopía.

\section{REFERENCIAS}

[1] Smith, W. F. Fundamentos de la Ciencia e Ingeniería de Materiales, Edit.: McGraw Hill. España, 1998.

[2] Gere, J. M. Mecánica de Materiales, Edit.: Thomson. México, 2002.

[3] ASTM International, Standard Test Methods for Testing of Metallic Materials. ASTME8/E8M-09. USA, 2005.

[4] Zienkiewicz, O. C. Finite Elements and Aproximation. Edit. : John Wiley \& Sons. Canada, 1983.

[5] Nicholson, D. W. Finite Element Analysis. Thermomechanism of solids. Edit. : CRC Press. USA, 2003. 